\title{
Integrating new information with old knowledge
}

\author{
CHARLES CLIFTON, JR. and MARIA L. SLOWIACZEK \\ University of Massachusetts, Amherst, Massachusetts 01003
}

\begin{abstract}
Three experiments investigated the integration of new information with old concepts about famous people and the extent to which learning conditions influence this integration. In Experiments 1 and 2, subjects learned a set of facts about famous people in a list or in the context of a short biography. The facts were normatively related to prior knowledge of the person. Subjects were tested on sentences that required them to make an inference combining the newly learned fact with a well-known old fact (e.g.. subjects learned "Walt Disney grew up on a farm" and were tested with "The creator of Mickey Mouse grew up on a farm "). They were faster when the newly learned facts were clearly related to old knowledge about the person, but only in the biography condition. In Experiment 3, inferences were verified faster when the description and fact were consistent with the same perspective, but. only in the story condition, not in the list condition. These experiments suggest that activating old knowledge facilitates integration and that new information is not necessarily integrated with all old knowledge about a given concept but, rather, is organized within a subconcept.
\end{abstract}

Our ability to understand and remember new information critically depends upon what we already know and how our knowledge is organized. Both common experience and ingenious experimental demonstrations (e.g., Bransford \& Johnson, 1972) tell us that we must succeed in relating new information to old knowledge if we are to understand and remember it. We will say information is "integrated" when it has been successfully related to old knowledge and remembered in terms of the relations that were found. In the research reported here, we studied how people learn new, previously unknown, facts about famous people, such as, "Mick Jagger was convicted of a drug offense." We varied how easily the facts could be related to old knowledge about the famous people, and we varied the context in which the new facts were learned, with the goal of identifying when new information would be integrated with old. We also addressed the question of whether new information that was integrated with old knowledge could easily be accessed from all old knowledge about the individual or only from old knowledge about the famous person that could be thematically related to the new information.

In order to address these questions, we measured how quickly our subjects could perform inferences that related new knowledge to old. Other researchers interested in the integration of information have used memorability (Bransford \& McCarrell, 1974; Schustack

This research was supported in part by a Faculty Research Grant from the University of Massachusetts to the senior author. We would like to thank Patrick Carroll, Rachel Clifton, and Jerome Myers for helpful critical readings of an earlier version of this paper. Requests for reprints should be sent to Charles Clifton, Department of Psychology, University of Massachusetts, Amherst, Massachusetts 01003 .
\& Anderson, 1979; cf. Cermak \& Craik, 1979) and protection from interference (Moeser, 1979; Smith, Adams, \& Schorr, 1978) as measures of integration. We assumed that an inference that requires combining new knowledge with old should be faster if the new knowledge had already been successfully related to some relevant old knowledge than if each were in a separate knowledge structure. Anderson (1977) and Anderson and Hastie (1974) have provided evidence that reaction time to verify inferences is less when one can reasonably expect integration to have occurred than when integration is unlikely. Further, Dooling and his associates (Dooling \& Christiaansen, 1977; Dooling \& Lachman, 1971; Sulin \& Dooling, 1974; cf. Schustack \& Anderson, 1979) and Moeser (1977) have shown that recognition of inferences is more likely after learning conditions that encouraged integration. Finally, it is possible to construct simple and plausible theoretical rationales for why inferences should be made rapidly after integration has occurred.

One illustrative theoretical formulation, designed to be broadly consistent with a network theory of semantic representation (Anderson, 1976; Anderson \& Bower, 1973 ) is shown in Figure 1. One fact associated with the concept node is the name of the individual specified by the concept ("Mick Jagger"), and another is a wellknown fact about that individual ("is the lead singer of the Rolling Stones"). A person who learns a statement asserting some new fact of the name Mick Jagger (e.g., "Mick Jagger was convicted of a drug offense") may manage to integrate that fact with old knowledge (such as the belief that rock singers' practices involving drugs are apt to be frowned upon by authority figures). Such integration can be represented by attaching the new fact directly to the node representing the concept 


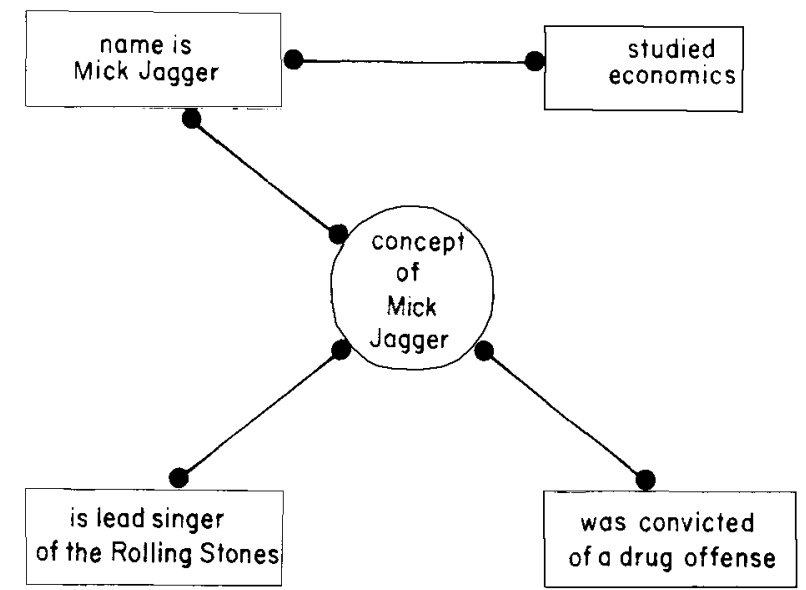

Figure 1. Network representation of integrated and uninte grated knowledge.

of Mick Jagger. If a new fact asserted of the name "Mick Jagger" (e.g., "studied economics") is not successfully integrated with previous knowledge, it is attached instead to the node representing the name "Mick Jagger." To verify the inferential statement "The lead singer of the Rolling Stones was convicted of a drug offense," two associations must be examined: the association between the subject phrase of the statement and the concept node and the association between the predicate of the statement and the concept node. To verify the inference "The lead singer of the Rolling Stones studied economics," three associations must be examined: subject to concept, predicate to name, and name to concept. Examining more associations should take more time.

In the experiments we report here, subjects learned new true statements about well-known individuals, referred to by their proper names. We measured how rapidly subjects could verify inferences made by replacing the proper name with a commonly known definite description of the person. We will refer to the difference in time needed to verify these two types of items as inference time. We used the measure of inference time to assess subjects' success in integrating new statements with old knowledge. The statements varied in the ease with which they could plausibly be related to old knowledge about the individual. We expected integration to be more successful for statements that could be more easily related to old knowledge. Learning conditions also varied. We expected that learning facts in the context of a biography or story would more successfully promote integration than learning them in a list. Finally, the facts used in Experiment 3 differed in whether they seemed thematically appropriate to one or another contrasting points of view about the famous people (e.g., Mick Jagger as rock star vs. Mick Jagger as family man). We wanted to learn whether integration could be specific to one point of view about a person.

\section{Normative Studies}

In the experiments to be reported, subjects learned previously unknown but true facts about famous people.
Three sets of norms were collected. The "knowledge norms" assessed how familiar the facts were to students of the University of Massachusetts. The "relatedness norms" measured how well a fact fit with prior knowledge about a famous person designated by name. The "appropriateness norms" measure how appropriate each fact was to each of two well-known definite descriptions of the famous people.

One group of 26 subjects provided data for the knowledge norms. These students were given 186 pairs of sentences, one true and one false (as determined by biographies and encyclopedias), and were asked to check which sentence was true. Each sentence made some assertion about one of nine famous people (Mick Jagger, Abraham Lincoln, Mark Twain, Walt Disney, Adolf Hitler, Richard Nixon, Elvis Presley, Jimmy Carter, and John Kennedy). The false sentences were closely matched in form to the true ones (e.g., true: "Richard Nixon went bankrupt selling frozen orange juice"; false: "Richard Nixon went bankrupt selling aluminum siding"). No fact that more than $70 \%$ of our subjects chose correctly was used in any experiment. The facts used in Experiments 1 and 2 were chosen correctly, on the average, $42.5 \%$ of the time, whereas the facts used in Experiment 3 were chosen $43.6 \%$ of the time. (Full normative data are available in Clifton and Slowiaczek, Note 1.)

Twenty-five other students provided the relatedness norms data. These subjects were given the predicate of each true sentence used in the knowledge norms, with a blank in the place of the subject. They were asked to fill in the blank with the name of the correct famous person chosen from the list of nine names. Relatedness scores were calculated by taking the percentage of subjects who chose the correct famous person as most compatible with a given fact. A fact that seemed to fit with a particular famous person and could thus be integrated with other knowledge about that person would receive a high relatedness score.

A third group of 47 subjects provided the appropriateness norms data, which were used in selecting materials for Experiment 3. They received a separate data sheet for each of the nine famous people. Two definite descriptions of the person (see the Appendix for those used in the critical conditions of Experiment 3) appeared at the top of the sheet, followed by the predicates of most of the unknown facts identified in the knowledge norms. The two definite descriptions were intuitively chosen to contrast thematically (as "lead singer of the Rolling Stones" and "husband of Bianca" seem to contrast as descriptions of Mick Jagger). Subjects rated how appropriate each definite description was for each fact, using a scale from 1 (highly appropriate) to 5 (highly inappropriate).

\section{EXPERIMENTS 1 AND 2}

The first two experiments were identical, except that subjects learned statements in the context of a capsule biography in Experiment 1 and as a list in 
Experiment 2. ${ }^{1}$ The experiments compared assertions that were presumably easy to integrate ("related" statements), as determined by high scores on the relatedness norms, with those that were difficult to integrate ("unrelated"' statements). The statements were intermixed in a biography in Experiment 1 and presented on separate lists in Experiment 2 . In order to encourage Experiment 2 subjects to integrate the related statements with prior knowledge, they were told that the related statements were in fact true and that the unrelated statements were fictional. In both experiments, inference time should be faster for facts that are successfully integrated than for those that are not. Related facts should be integrated more successfully than unrelated facts, but integration may be successful only in the context of a biography that activates old knowledge with which the new facts can be integrated.

\section{Method}

Subjects. Sixteen undergraduates served as subjects in Experiment 1 , and 16 other undergraduates served in Experiment 2.

Materials. Four related and four unrelated statements (as defined by the relatedness norms) for each of six famous people were chosen as test statements. In addition, two related and two unrelated statements were chosen for two other famous people and used for practice. The mean relatedness score was $73 \%$ for the related statements and $18 \%$ for the unrelated statements. All 56 facts were identified as "unknown" to our subjects in the knowledge norms.

For Experiment 1, a capsule biography was made for each of the famous people. Each biography included the eight facts about the famous person (four for the practice people) explicitly mentioning the person's proper name (e.g., "Elvis Presley collected teddy bears"). Filler sentences about the person were used to connect the test sentences to a story.

For Experiment 2, the 24 related test and 4 related practice statements were presented as one list of 28 facts, and the 28 unrelated statements were presented as a separate list. The statements appeared exactly as they did in the biographies used in Experiment 1.

Two well-known descriptions about each person were used to make inference tests for both experiments. The descriptions involved no information that was directly stated in the biographies and were never shown to subjects before they appeared on the test trials. The unknown facts and the descriptions were similar to those presented in the Appendix, the materials for Experiment 3.

Procedure. Subjects in Experiment 1 were told to take as much time as they needed to learn the material in the eight capsule biographies. After a subject indicated that the material was learned (typically after about $25 \mathrm{~min}$, for both Experiments 1 and 2), a training drill was given. The predicate of each statement was presented individually, and the subject was asked to identify it by naming the correct person. The drill continued until all statements had been identified correctly.

The training procedure was identical for Experiment 2, except that subjects were told to study the two lists of 28 statements. They were told that the related statements were true and were asked to associate them with other things that they knew. They were told that the unrelated statements were fictional, but were encouraged to study them carefully. In the training drill, the subjects were required to identify each fact as "true" or "fictional," in addition to identifying the person about whom the fact was learned.

Immediately after the training drill, subjects were given a verification test of 16 practice trials and four blocks of 24 test trials. On each trial, a single sentence was presented on a
Table 1

Test Types Used in Experiments 1 and 2

\begin{tabular}{ll}
\hline N-R & $\begin{array}{l}\text { Mick Jagger was convicted of a drug offense. } \\
\text { D-R }\end{array}$ \\
The lead singer of the Rolling Stones was convicted \\
of a drug offense.
\end{tabular}

computer-controlled video monitor for verification. There were four types of test sentences: N-R, a name of a famous person with a predicate of a related statement; D-R, a description of a famous person with the predicate of a related statement; $\mathrm{N}-\mathrm{U}$, a name with the predicate of an unrelated statement; and $\mathrm{D}-\mathrm{U}$, a description with the predicate of an unrelated statement. An example of the test types is presented in Table 1 . Half the tests were negative trials, in that the name or description was presented with the predicate of a statement that had been learned about a different person. Subjects pulled one lever to indicate that the name or description was correctly paired with the predicate and another lever to indicate a mispairing. They were instructed to ignore the true vs. fictitious distinction. Reaction time was measured, and subjects were given immediate feedback when they made an error. Feedback about average reaction time and total number of errors was provided at the end of each trial block.

Design. On the practice trials, only the names, descriptions, and statements of the two practice people were used. Each practice person was tested eight times, equally divided between positive and negative and related and unrelated statements. In Experimental Block 1, each of the six famous people was tested on a positive trial for two test types and a negative trial for the other two test types. These assignments were reversed for Block 2, so that over these two blocks all of the famous people were tested on a positive and a negative trial for each of the four test types. Trial assignments were balanced over famous people and experimental block so that an equal number of positive/ negative, name/description, and related/unrelated tests were presented in each block. Blocks 3 and 4 repeated the design of Blocks 1 and 2, except that the specific predicates that were presented on negative trials were presented on positive trials and vice versa. Across subjects, each famous person and each predicate appeared equally of ten in each test condition on each trial block, and the order of trials within each block was randomized independently for each subject.

\section{Results}

Experiment 1. The mean reaction times for correct positive responses are presented in Table 2. Means for Block 1 are presented separately, since subjects first saw the definite descriptions on this block. However, since they were consistent with the overall data, only the latter were analyzed statistically. Inference time (description reaction time minus name reaction time) was less for related statements than for unrelated statements [729 vs. $924 \mathrm{msec}, \mathrm{F}(1,15)=5.06, \mathrm{p}<.05$ ] Related statements were verified faster overall than unrelated statements $[2,316$ vs. $2,403 \mathrm{msec}, F(1,15)=$ $5.00, \mathrm{p}<.05]$. Further, name tests were faster than description tests $[1,946$ vs. $2,773 \mathrm{msec}, F(1,15)=190.0$, $\mathrm{p}<.001]$.

Error rates are presented in Table 3. They were higher for the unrelated than for the related statements, which 
Table 2

Mean Verification Times for Correct Positive Responses: Experiments 1 and 2

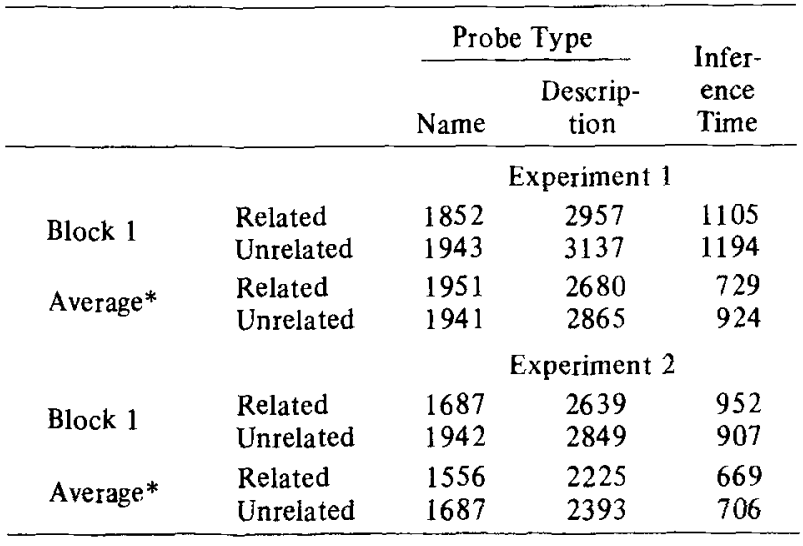

*All blocks.

Table 3

Error Percentages: Experiments 1 and 2

\begin{tabular}{clcc}
\hline & & \multicolumn{2}{c}{ Probe Type } \\
\cline { 3 - 3 } Response & & Name & Description \\
\hline \multirow{3}{*}{ Positive } & Related & 6 & 15 \\
& Unrelated & 13 & 22 \\
Negative & Related & 4 & 5 \\
& Unrelated & 8 & 9 \\
& & Experiment 2 \\
Positive & Related & 3 & 7 \\
& Unrelated & 10 & 20 \\
Negative & Related & 6 & 6 \\
& Unrelated & 9 & 15 \\
\hline
\end{tabular}

could indicate that the related statements were learned better.

Experiment 2. Reaction times for Experiment 2 correct positive responses also appear in Table 2. Inference time was nonsignificantly larger for the related than for the unrelated statements on Block 1 (952 vs. $907 \mathrm{msec}$ ). The difference reversed, still nonsignificantly, when the data were averaged over all blocks. Response time was faster for related statements than for unrelated statements, averaged over both the name and the description tests. Although this may indicate that the related statements were learned better than the unrelated ones, there is no evidence that the related statements were organized differently in memory than the unrelated ones.

The error rates, which appear in Table 3 , are generally similar to those in Experiment 1. They were higher for unrelated than for related statements, suggesting a higher degree of learning of the related statements.

\section{Discussion}

Inference time for statements that were easily related to old knowledge was less than that for unrelated statements when the statements had been learned in the context of a biography. When the statements had been learned as a list, subjects were told that the related statements were true and that the unrelated statements were fictitious to encourage them to distinguish between related and unrelated statements. Nonetheless, we found no difference in inference time in the list experiment. We suggest that learning the facts in a biography activated old knowledge with which the new information could be successfully integrated, whereas learning the facts in a list did not. However, even in the context of a biography, integration was successful only for those new facts that were easily related to old knowledge.

A close examination of the size of the inference effect obtained for each statement indicated that new information, when integrated, was integrated best with old knowledge to which it was thematically related. Based on subjective judgment, we divided the related statements into two groups. The "congruent" group included all the description-statement pairs in which the description and the predicate of the statement seemed to be related to the same perspective (schema, point of view) about the famous person. For instance, we judged "The lead singer of the Rolling Stones" and "was given a prison sentence for a drug offense" to be related to the same perspective of Mick Jagger. The "incongruent" group included all the descriptionstatement pairs in which the description and the predicate of the statement was related to a different perspective. "The husband of Bianca" and "was given a prison sentence for a drug offense" constitute a pair that belongs to this group. Inference time in Experiment 1 was $623 \mathrm{msec}$ for the congruent pairs and $882 \mathrm{msec}$ for the incongruent pairs.

This difference suggests that new information is integrated, not with a general concept of an individual but with a single point of view about that individual, or even with particular pieces of old knowledge about that individual. However, the difference found in this post hoc analysis is only suggestive and must be confirmed in a properly designed experiment. This was done in Experiment 3.

\section{EXPERIMENT 3}

\section{Method}

Subjects. Forty-eight undergraduates participated as subjects. Each was assigned to one of three experimental groups of equal size: list, Story A, and Story B. One subject in the list group was discarded after the experiment was completed, when it was discovered that his error rate was $43 \%$ on the last block of trials and $16 \%$ overall.

Materials. Four well-known definite descriptions were constructed for each of four famous people. Descriptions A and B represented contrasting points of view about the individual and were used on the positive test trials. The other two descriptions were used only on practice and negative test trials.

Two minibiographical stories were written about each person. The stories did not mention any of the definite descriptions. They can be found in Clifton and Slowiaczek (Note 1).

Seven unknown facts (according to our knowledge norms) were chosen for cach famous person. Four were to be used as positive test items, and three as negative and practice test items. 
Two facts for each person, referred to as Fact A facts, were thematically consistent with Description A, according to our appropriateness norms, and the two Fact $B$ facts were thematically consistent with Description $B .^{2}$

Descriptions A and B and the two Fact A and two Fact B facts for each person appear in Appendix 1. The appropriateness scores of each fact judged against each description also appear in Appendix 1; a 1 indicates a judgment of high appropriateness and a 5 a judgment of low appropriateness. The relatedness scores of these facts (the normative data used to select facts for Experiments 1 and 2) varied greatly among facts but averaged $59 \%$.

Procedure. Subjects in the story groups were asked to learn the statements in the set of stories they read. Subjects in the list group were asked to learn a list of 56 statements (7 statements about each of the four famous people for whom data are reported, plus four other people; see Footnote 2). They were also asked to write a continuation of each statement to aid memory. Study was self-paced and generally took about $20 \mathrm{~min}$. After the study period, subjects were drilled until they were able to identify about whom each fact had been asserted.

The verification test consisted of eight blocks of 14 trials after one practice block of 16 trials. On each trial, a single sentence was presented for verification on a computer-controlled video terminal. The positive test sentences consisted of the name of a famous person, or one of the A or B descriptions of the person together with the predicate of a newly learned statement about the person. Negative test sentences were constructed by mispairing names or $\mathrm{C}$ or $\mathrm{D}$ descriptions with predicates. Reaction time was measured and feedback given, as in Experiments 1 and 2 .

Design. Two statements about each famous person were presented on the practice trials, with an equal number of positive and negative and name and description trials. Only the three practice and negative facts about each person and the $C$ and $D$ descriptions were used in practice.

After the practice trials, 14 statements about each famous person were presented. Eight statements required a positive response, and six a negative response. There were three types of positive tests. Proper name tests paired a name with an $A$ fact (N-Fact A) or a B fact (N-Fact B). Consistent tests paired a fact and a description that fit with the same point of view (DA-Fact A, DB-Fact B). Inconsistent tests paired a description from one point of view with a fact from the other point of view (DA-Fact B, DB-Fact A). During the first four trial blocks, each experimental subject received proper-name and consistent tests (both DA-Fact A and DB-Fact B) about two famous people, and proper-name and inconsistent tests about the other two famous people. The assignment of famous people to consistent vs. inconsistent tests and the assignment of facts to name and description tests were reversed during the last four trial blocks. Over all subjects, each famous person and each fact received each type of test equally often on each block of trials.

\section{Results}

Correct positive reaction times were analyzed, after discarding the $2.6 \%$ of responses that were longer than $3,500 \mathrm{msec}$. Since the two different story groups did not differ significantly, and since the first vs. the last four trial blocks did not interact significantly with any experimental variables, the mean reaction times were averaged over these variables and are presented in Table 4. Error rates are presented in Table 5 .

The critical interaction of Training Condition by Probe Type was significant $[\mathrm{F}(2,90)=5.83, \mathrm{p}<.01]$. Inference time for consistent tests was less than that for inconsistent tests when the facts had been learned in a story (366 vs. $472 \mathrm{msec}, \mathrm{p}<.01$ ). This pattern was
Table 4

Mean Reaction Times for Correct Positive Responses: Experiment 3

\begin{tabular}{lrr}
\hline & \multicolumn{2}{c}{ Training Condition } \\
\cline { 2 - 3 } \multicolumn{1}{c}{ Probe Type } & List & Story \\
\hline Name & 1638 & 1566 \\
Consistent Descriptions & 2047 & 1932 \\
Inconsistent Descriptions & 1962 & 2038 \\
Consistent Inference Time CD-N & 409 & 366 \\
Inconsistent Inference Time ID-N & 324 & 472 \\
\hline
\end{tabular}

Table 5

Error Percentages for Positive Test Trials: Experiment 3, Averaged Over All Blocks

\begin{tabular}{lcc}
\hline & \multicolumn{2}{c}{ Training Condition } \\
\cline { 2 - 3 } & List & Story \\
\hline PN & 13 & 14 \\
Consistent & 5 & 6 \\
Inconsistent & 8 & 11 \\
\hline
\end{tabular}

found for each of the four famous people tested. However, the opposite held true when the facts had been learned in a list ( 409 vs. $324 \mathrm{msec}, \mathrm{p}<.05$ ); this pattern was found for three of the famous people tested, with no difference in the fourth case. Learning facts in a story as opposed to a list speeded response time for name tests (by $72 \mathrm{msec}, .10>p>.05$ ) and for consistent tests $(115 \mathrm{msec}, \mathrm{p}<.01)$ but slowed response time for inconsistent tests $(76 \mathrm{msec}, \mathrm{p} \simeq .05)$. As always, name probes were faster than description probes $[F(2,90)=118, p<.01]$.

\section{Discussion}

Experiment 3 confirmed the findings of Experiments 1 and 2, including the post hoc analysis of Experiment 1. The way in which new facts are organized with old knowledge seems to be critically dependent on the context in which they are learned. Response times to verify inference sentences showed that when facts were learned within a story, they were integrated with old knowledge that represented a similar perspective. The contrast between consistent and inconsistent tests showed that even though a fact was integrated with some old knowledge about a particular person, that fact was not easily retrievable from all old knowledge about that person. This finding can be seen as an instance of the phenomenon of encoding specificity (Tulving \& Thomson, 1973) operating in the realm of knowledge about real individuals.

The finding that inconsistent test sentences received faster response times than consistent tests in the list condition is surprising and deserves examination. We might have expected faster response times for consistent test items even without integration. The obtained result might reflect an attempt on the part of our subjects to integrate the facts in the list condition. Recall that in the Experiment 3 list condition, unlike in Experiment 2, subjects were instructed to write continuations to all statements and to try to make sense out of them. 
Perhaps subjects tried to integrate all the facts with their dominant point of view of the person. Subjects might have to put more effort into associating the inconsistent facts with the dominant point of view, resulting in better performance on the inconsistent tests. This line of reasoning leads us to expect the dominant description to be responsible for most of the difference between consistent and inconsistent tests in the list condition. A post hoc look at the data, in which we intuitively classified the descriptions into those that related to the culturally dominant point of view about a famous person and those that did not, indicated that this was the case. Dominant descriptions were $124 \mathrm{msec}$ faster for inconsistent tests than for consistent tests in the list condition. Nondominant descriptions were only $46 \mathrm{msec}$ faster for inconsistent tests than for consistent tests.

\section{GENERAL DISCUSSION}

The time taken to verify inferences points to some conclusions about how new knowledge is integrated with old. Experimental subjects who learned new facts about famous people in the context of a story seemed to organize the new information with old knowledge in an orderly fashion. In Experiment 1, they verified inferences faster when the new facts were already related to old knowledge than when they were not. In Experiment 3, they verified inferences rapidly when the old and new facts combined in the inference were related to the same perspective about the famous person; they were not fast when the facts were related to different perspectives.

When the statements were presented as a list, subjects seemed to have more freedom in how they could organize the facts. In Experiment 2, in which subjects were told that some of the statements were false, inference times showed no indication of integrative organization. In Experiment 3, in which subjects were instructed to relate new knowledge to old, they seemed to try to incorporate all facts with their more dominant point of view about the famous person.

The initial versions of current network models of memory (e.g., Anderson \& Bower, 1973) suggested that information about an individual is associated with a single general concept of that individual. Anderson (1977), Anderson and Hastie (1974), and Anderson and Paulson (1978) have shown that information learned about one description of an individual does not fully generalize to other descriptions of the same individual. Reder and Anderson (1980) found that events learned from one episode interfered less with recognition of events from a different episode than they did with recognition of events from the same episode. Anderson and Pichert (1978) found that the perspective from which a story was recalled affected what facts were remembered. They found that recall was higher for facts related to the point of view taken at recall time than for facts not so related. This finding is conceptually equivalent to ours, except that it deals with recall accuracy rather than with inference time. All these phenomena indicate that facts about a given individual can be stored with distinct subconcepts of that individual, hindering some types of memory retrieval and facilitating others. Reder and Anderson (1980) make a conceptually equivalent claim in their "subnodes" model. In terms of our Fig. ure 1, the distinct subconcepts model could be expressed by adding separate subnodes corresponding to Mick Jagger as rock star and as student, to which the appropriate facts could be attached.

The present experiments go beyond previous research in indicating that distinct subconcepts exist for old welllearned knowledge, as well as for facts memorized for a psychological experiment. They indicate that, when new information is integrated with old knowledge about a topic, it is integrated with a specific domain of that old knowledge. New information can be accessed rapidly from other pieces of information within that domain, but has no particular advantage in how rapidly it is accessed from old knowledge outside that domain.

\section{REFERENCE NOTE}

1. Clifton, C., Jr., \& Slowiaczek, M. L. Integrating knowledge: George Washington on both sides of the coin (Tech. Rep. 79-1). Amherst: Cognitive Processes Laboratory, University of Massachusetts, June 1979

\section{REFERENCES}

ANDERSON, J. R. Language, memory and thought. Hillsdale, N.J: Erlbaum, 1976.

Anderson, J. R. Memory for information about individuals. Memory \& Cognition, 1977, 5, 430-442.

Anderson, J. R., \& Bower, G. H. Human associative memory. Washington, D.C: Winston, 1973.

Anderson, J. R., \& Hastie, R. Individuation and reference in memory: Proper names and definite descriptions. Cognitive Psychology, 1974, 6, 495-514.

Anderson, J. R., \& Paulson, R. Interference in memory for pictorial information. Cognitive Psychology, 1978, 10, 178-202.

Ande rson, R. C., \& Pichert, J. V. Recall of previously unrecallable information following a shift in perspective. Journal of Verbal Learning and Verbal Behavior, 1978, 17, 1-12.

Bransford, J. D., \& Johnson, M. K. Contextual prerequisites for understanding: Some investigations of comprehension and recall. Journal of Verbal Learning and Verbal Behavior, 1972, 11, 717-726.

Bransford, J. D., \& McCarrell, N. S. A sketch of a cognitive approach to comprehension: Some thoughts about understanding what it means to comprehend. In W. B. Weimer \& D. S. Palermo (Eds.), Cognition and the symbolic processes. Hillsdale, N.J: Erlbaum, 1974.

Cermak, L. S., \& Craik, F. (Eds.). Levels of processing in human memory. Hillsdale, N.J: Erlbaum, 1979.

Dooling, D. J., \& Christiannsen, R. E. Episodic and semantic aspects of memory for prose. Journal of Experimental Psychology: Human Learning and Memory, 1977, 3, 428-436.

Dooling, D. J., \& Lachman, R. Effects of comprehension on retention of prose. Journal of Experimental Psychology, $1971,88,216-222$.

Moeser, S. D. Recognition processes in episodic memory. Canadian Journal of Psychology, 1977, 31, 41-70.

Moesfr. S. D. The role of experimental design in investigations of the fan effect. Journal of Experimental Psychology: Human Learning and Metnory, 1979, 5, 125-134.

Reder, L. M., \& ANDerson, J. R. A partial resolution of the paradox of interference: The role of integrating knowledge. Cognitive Psychology, 1980, 12, 447-472. 
Schustack, M. W., \& Anderson, J. R. Effects of analogy to prior knowledge on memory for new information. Journal of Verbal Learning and Verbal Behavior, 1979, 18, 565-583.

Smith, E. E., Adams, N., \& Schorr, D. Fact retrieval and the paradox of interference. Cognitive Psychology, 1978, 10, 438-474.

Sulin, R. A., \& Dooling, D. J. Intrusion of a thematic idea on retention of prose. Journal of Experimental Psychology, $1974,103,255-262$.

Tulvina, E., \& Thomson, D. M. Encoding specificity and retrieval operations in episodic memory. Psychological Review, $1973,80,352-373$.

\section{NOTES}

1. Ideally, Experiments 1 and 2 would have been conducted as a single experiment and could be reported as such. However, the experiments were performed sequentially, at different times in the academic semester, and we fear that somewhat different subject populations were represented in them. The substantial differences in overall reaction time between the experiments may be due to that or to other factors, such as the higher degree of learning in Experiment 2 because of its more elaborate training drill or the learning of irrelevant and untested facts in the biographies of Experiment 1 . Interpretive difficulties are largely removed by the results of Experiment 3 , in which lists and biography conditions were compared within a single experiment.

2. Names of four additional famous people were also used in the experiment, but data from these tests of facts learned about them are not reported here. We were not able to select materials from our norms that represented two distinct perspectives on these famous people. An attempt was made to write the two stories for each of the main four people to represent different points of view, to test a hypothesis that point of view under which a fact was learned would influence how it was integrated. However, we were not successful in writing stories that presented distinct points of view clearly enough. Full details appear in Clifton and Slowiaczek (Note 1).

Appendix 1

Materials Used in Experiment 3

\begin{tabular}{|c|c|c|c|}
\hline \multirow[b]{2}{*}{ Description } & \multirow[b]{2}{*}{ Fact } & \multicolumn{2}{|c|}{$\begin{array}{c}\text { Appropriate- } \\
\text { ness Rating }\end{array}$} \\
\hline & & $A^{*}$ & $\mathrm{~B} \dagger$ \\
\hline \multicolumn{4}{|l|}{ Mick Jagger } \\
\hline A: The husband of Bianca & A: Has a daughter named Jade & 1.85 & 3.62 \\
\hline \multirow[t]{3}{*}{ B: The lead singer of the Rolling Stones } & A: Was married in St. Tropez & 1.64 & 3.77 \\
\hline & B: Was given a prison sentence for a drug offense & 3.74 & 2.47 \\
\hline & B: Recited Shelley at a concert in London & 4.13 & 2.57 \\
\hline \multicolumn{4}{|l|}{ Abraham Lincoln } \\
\hline A: The President who freed the slaves & A: Failed in his first try for elective office & 2.83 & 3.39 \\
\hline \multirow[t]{3}{*}{ B: The man who grew up in a log cabin } & A: Belonged to the Whig party when first elected to office & 2.55 & 3.69 \\
\hline & B: Lived on a farm on Knob Creek & 4.23 & 1.96 \\
\hline & B: Earned his first money by ferrying people on the Ohio River & 4.10 & 2.50 \\
\hline \multicolumn{4}{|l|}{ Mark Twain } \\
\hline A: The author of Tom Sawyer & A: Designed his home after a Mississippi steamboat & 2.25 & 3.94 \\
\hline \multirow[t]{2}{*}{ B: The satirist and famous lecturer } & $\begin{array}{l}\text { A: Signed his early stories "Josh" } \\
\text { B: Gave a financially successful world lecture tour }\end{array}$ & $\begin{array}{l}2.69 \\
2.90\end{array}$ & $\begin{array}{l}3.25 \\
1.90\end{array}$ \\
\hline & B: Made frequent trips to Europe & 3.72 & 2.33 \\
\hline \multirow{4}{*}{$\begin{array}{l}\text { Walt Disney } \\
\text { A: The producer of nature films } \\
\text { B: The creator of Mickey Mouse }\end{array}$} & & & \\
\hline & $\begin{array}{l}\text { A: Made live action films becau } \\
\text { A: Grew up on a farm }\end{array}$ & $\begin{array}{l}2.13 \\
1.83\end{array}$ & $\begin{array}{l}3.96 \\
3.38\end{array}$ \\
\hline & B: Used his hometown streets as a model for Main Street in Disneyland & 3.50 & 3.19 \\
\hline & B: Started a company of animated films named Laugh-O-Grams & 3.29 & 1.85 \\
\hline
\end{tabular}

*Description $A . \quad$ fDescription $B$.

(Received for publication April 9, 1980; revision accepted July 25,1980 .) 